\title{
Analysis of the Three-Dimensional Dynamic Problems by Using a New Numerical Method
}

\author{
Yao Rong $\mathbb{D},{ }^{1}$ Yang Sun, ${ }^{1}$ LiQing Zhu, ${ }^{1}$ and Xiao Xiao $\mathbb{D}^{2}$ \\ ${ }^{1}$ Jiangxi Transportation Research Institute, Nanchang 330200, China \\ ${ }^{2}$ Jiangxi Provincial Engineering Research Center of the Special Reinforcement and Safety Monitoring \\ Technology in Hydraulic \& Civil Engineering, Nanchang 330099, China \\ Correspondence should be addressed to Xiao Xiao; xiaoxiao8808@163.com
}

Received 20 February 2021; Revised 22 March 2021; Accepted 20 April 2021; Published 5 May 2021

Academic Editor: Dongliang Mei Zhang

Copyright (C) 2021 Yao Rong et al. This is an open access article distributed under the Creative Commons Attribution License, which permits unrestricted use, distribution, and reproduction in any medium, provided the original work is properly cited.

\begin{abstract}
The problems of the consolidation of saturated soil under dynamic loading are very complex. At present, numerical methods are widely used in the research. However, some traditional methods, such as the finite element method, involve more degrees of freedom, resulting in low computational efficiency. In this paper, the scaled boundary element method (SBFEM) is used to analyze the displacement and pore pressure response of saturated soil due to consolidation under dynamic load. The partial differential equations of linear problems are transformed into ordinary differential equations and solved along the radial direction. The coefficients in the equations are determined by approximate finite elements on the circumference. As a semianalytical method, the application of scaled boundary element method in soil-structure interaction is extended. Dealing with complex structures and structural nonlinearity, it can simulate two-phase saturated soil-structure dynamic interaction in infinite and finite domain, which has an important engineering practical value. Through the research, some conclusions are obtained. The dimension of the analytical problem can be reduced by one dimension if only the boundary surface is discretized. The SBFEM can automatically satisfy the radiation conditions at infinite distances. The 3D scaled boundary finite element equation for dynamic consolidation of saturated soils is not only accurate in finite element sense but also convenient in mathematical processing.
\end{abstract}

\section{Introduction}

Dynamic consolidation analysis of foundation is one of the most concerned problems, such as seismic design of large underground caverns, high arch dams, super high-rise buildings, and other large-scale industrial and their response characteristics under mechanical vibration (Chen and $\mathrm{He}$ [1], Lu et al. [2], and Jiang and Liang [3]).The free field formed by dynamic loads not only affects the dynamic response of long-span structures but also has feedback effect on the propagation of vibration waves by different components. Therefore, it is not reasonable to study the structure-constrained system as an isolator. It is necessary to consider the dynamic interaction between structure and foundation soil. For foundation soils in semi-infinite space, many literatures regard foundation soils as single-phase materials. When the foundation soil with high water content is liquefied by earthquake load, the pore pressure will increase with time, which will have a greater impact on the safety of the structure (Du and Wang [4] and Xia et al. [5]). Therefore, considering that the foundation soil is saturated soil with two-phase coupling of water and soil, the analysis of dynamic consolidation can better reflect the engineering practice.

For infinite and semi-infinite foundation soils, the main numerical analysis methods are finite element method (FEM), boundary element method, and finite elementboundary element coupling method. The simulation of semiinfinite foundation soil is much more complicated compared to the finite element method mainly because of the large discrete range of foundation soil. In order to obtain enough calculation accuracy, the free degrees of the system increased inevitably. Although the computing power has been greatly improved, the computational units needed for some special 
cases, such as the accurate analysis of $3 \mathrm{D}$ stress wave propagation in anisotropic soils, are still difficult to achieve. Generally, the finite element discrete analysis is used to study foundations around the structure. Free or fixed boundary conditions are used to simulate infinite foundation soil. At the same time, in order to eliminate false reflection on artificial boundary, a transmission boundary model is established, for example, viscous and viscoelastic boundary (Zhang [6]), superposition boundary (Smith [7]), paraxial boundary (Engquist and Majda [8]), transient transmission boundary (Liao et al. [9] and Liao et al. [10]), and multidirectional and bi-asymptotic multidirectional transmission boundary (Keys [11] and Wolf and Song [12]). The main problems of finite element method based on the infinite boundary model, when it is used to analyze the dynamic consolidation of infinite foundation soil, are the inadequate accuracy of low-order boundary and the poor stability of high-order boundary. It does not have the accuracy in the sense of finite element. In other words, the numerical solution can converge to the solution when the discrete mesh is infinitely small.

The wave attenuation factor is introduced into the shape function to describe the propagation of vibration waves along infinite distances for the infinite element method (Zhao and Valliappan [13] and Khaliliet al [14]), which is also a numerical method for infinite domain problems. However, the accuracy depends largely on the selection of radial shape function. When the order of the radial shape function is low, the infinite element must be placed far away for higher accuracy, which will lead to the expansion of the solution scale of the system. The higher order radial shape functions usually leads to ill-conditioned equations. In addition, similar to the local transmission boundary, the infinite element method is not accurate enough in the finite element sense. In dealing with these problems of infinite and semi-infinite domains, the boundary element method could satisfy the radiation conditions very well at infinity, and there is no reflection problem of artificial boundary (Bonnet [15] and $\mathrm{Xu}$ et al. [16]). However, the basic solutions needed to form the integral equation of boundary element method are generally complex, especially for anisotropic materials, and there is not even a basic analytical solution. On the contrary, the treatment of singular integral or even hypersingular integral of boundary integral equation is very difficult, which limits the further application of boundary element method. The FEM-BEM coupling method has been widely used in the analysis of structure-foundation interaction (Yazdchiet al. [17] and Du [18]). However, it is difficult to coordinate the coupled deformation between FEM and BEM for the interface between structure and foundation.

Scaled boundary finite element method (SBFEM) is a boundary element method based on finite element method. Compared with the boundary element method, it does not need to solve the fundamental solution, so it can effectively deal with the problem of anisotropic media which is particularly complex and satisfies certain conditions. The scaled boundary finite element method (SBFEM) successfully satisfies Sommer field radiation condition by choosing appropriate similar centers. Therefore, the waves emitted by the source can only dissipate to infinity in the form of dewave, but not return from infinity in the infinite domain. At present, SBFEM has been applied to the analysis of wave problems in time domain and frequency domain and to the solution of boundary dynamic stiffness matrix of infinite foundation (Wolf and Song $[19,20]$ ). Previous results show that the SBFEM is very accurate and effective in dealing with most problems of infinite medium, anisotropic medium, and inhomogeneous change of materials. Considering that the foundation soil is a saturated soil with two-phase coupling of water and soil, the dynamic consolidation of 3D infinite saturated soil is analyzed by the SBFEM, which has not been reported in previous literatures.

\section{Governing Equation}

Taking 3D saturated soil area $V$ as the research object in Cartesian rectangular coordinates, the cross section is shown in Figure 1. If the field $V$ is a finite field, the scale curvilinear coordinate system shown in Figure 2 is established for a unit $V^{e}$ in the field, whose bottom surface is $S^{e}$ and the cone surface is $A^{e}$. For convenient of expression, the scaled center point $C$ coincides with the origin point $O$ of the Cartesian coordinate system. When the SBFEM is used, elements similar to the FEM are discretized on the $S^{e}$ boundary, which is on the bottom of the domain. $\eta$ and $\zeta$ are the toroidal local coordinates of the elements on the $S^{e}$ boundary in the surface coordinate system, and $\xi$ is the radial coordinates perpendicular to the boundary. $\eta, \zeta= \pm 1$ represents the edge of bottom $S^{e}$. Radial coordinate $0 \leq \xi \leq 1$. When $\xi=0$ or 1 , it represents the scale center point and the bottom of the finite field, respectively. The bottom $s$ of scaled center point and finite field $V$ are represented, respectively. When the position of the scaled center is determined, a one-to-one conversion relationship is formed between the Cartesian rectangular coordinate system and the local scaled boundary coordinate system, which is called the scaled boundary transformation.

For the 3D infinite saturated soil shown in Figure 1(b), the scaled center point is selected outside the infinite domain. Similar to the finite field, the vertex of the element cone is the scaled center point $C$, and the bottom represents the interface (as shown in Figure 2). Then, the radial coordinates $\xi \geq 1$ and $\xi=1$ correspond to the bottom of the infinite field. The whole infinite field is formed by assembling all the cones.

In the local coordinate system of the scaled boundary, any point on the bottom $S^{e}$ of $\xi=1$ can be expressed by $n$ node coordinates of the discrete element on the bottom as follows:

$$
\begin{aligned}
& x(\eta, \zeta)=[N(\eta, \zeta)]\{x\}=[N]\{x\}, \\
& y(\eta, \zeta)=[N(\eta, \zeta)]\{y\}=[N]\{y\}, \\
& z(\eta, \zeta)=[N(\eta, \zeta)]\{z\}=[N]\{z\},
\end{aligned}
$$

where the shape function is defined as $[N(\eta, \zeta)]=$

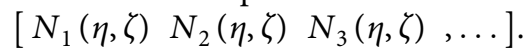




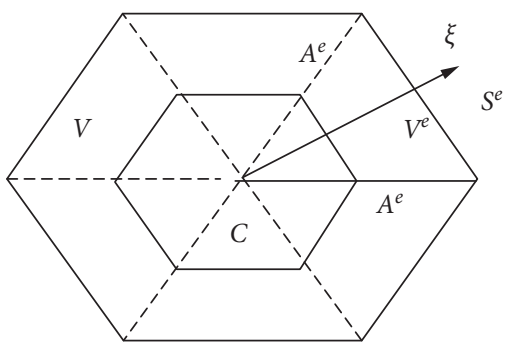

(a)

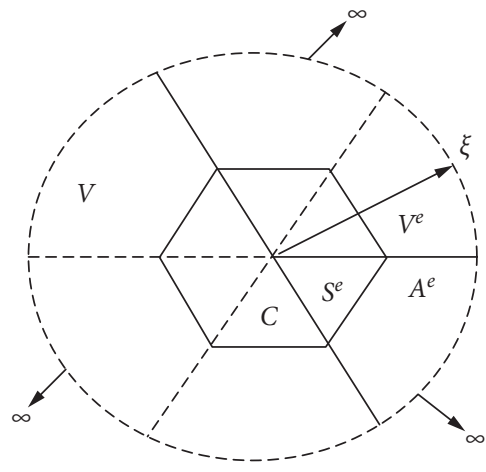

(b)

Figure 1: Cutaway view of three-dimensional saturated soil: (a) finite region and (b) unbounded media.

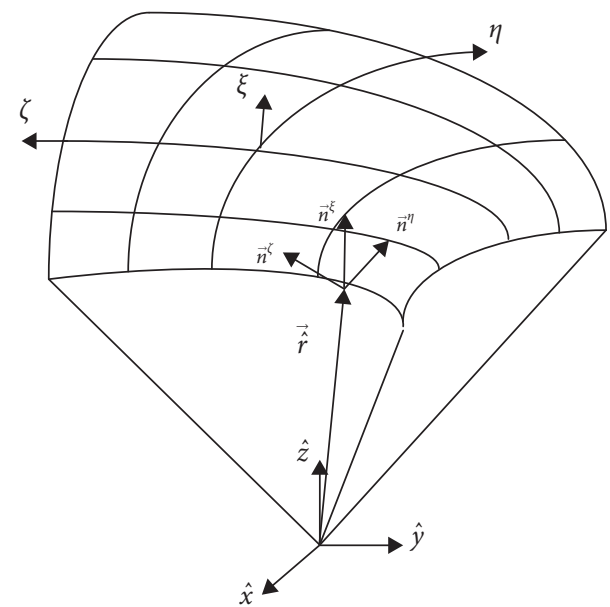

Figure 2: Transformation of global Cartesian coordinates to scaled boundary coordinates.

In the scaled coordinates, the relationship between point $\widehat{r}=\hat{x} i+\hat{y} j+\widehat{z} k$ in the computational element domain $V^{e}$ and bottom $S^{e}$ boundary point $r=x i+y j+z k$ can be expressed as follows:

$$
\begin{aligned}
& \hat{x}(\xi, \eta, \zeta)=\xi x(\eta, \zeta), \\
& \hat{y}(\xi, \eta, \zeta)=\xi y(\eta, \zeta), \\
& \widehat{z}(\xi, \eta, \zeta)=\xi z(\eta, \zeta) .
\end{aligned}
$$

Then,

$$
\left\{\begin{array}{c}
\frac{\partial}{\partial \widehat{x}} \\
\frac{\partial}{\partial \widehat{y}} \\
\frac{\partial}{\partial \widehat{z}}
\end{array}\right\}=[\widehat{J}]^{-1}\left\{\begin{array}{c}
\frac{\partial}{\partial \xi} \\
\frac{\partial}{\partial \eta} \\
\frac{\partial}{\partial \zeta}
\end{array}\right\}
$$

where Jacobian matrix $[\widehat{J}(\eta, \zeta)]=\left[\begin{array}{l}\hat{x}_{\xi}, \hat{y}_{\xi}, \hat{z}_{\xi}, \\ \hat{x}_{\eta}, \hat{y}_{\eta}, \widehat{z}_{\eta}, \\ \hat{x}_{\zeta}, \hat{y}_{\zeta}, \widehat{z}_{\zeta},\end{array}\right]$.
According to Biot's theory, the governing equations of consolidation of saturated soils include the dynamic equilibrium equation of soil skeleton and the continuous equation of fluid. In the Cartesian coordinate $\operatorname{system}(\hat{x}, \hat{y}, \widehat{z})$, the dynamic equilibrium equation expressed $_{T}$ by the displacement $(\{u\}=\{u(\widehat{x}, \widehat{y}, \widehat{z})\}=$ $\left.\left\{u_{x} u_{y} u_{z}\right\}^{T}\right)$ of soil skeleton in frequency domain is as follows:

$$
[L]^{T}\left(\left\{\sigma^{\prime}\right\}+\{m\} p_{f}\right)+\rho \omega^{2}\{u\}+\rho\{b\}=0,
$$

where $p_{f}$ is pore water pressure, $\omega$ is frequency, and $\rho=\rho_{s}+(1-\phi) \rho_{f} . \rho_{s}$ and $\rho_{f}$ are soil skeleton ${ }_{T}$ and fluid density, $\phi$ is porosity, $\{b\}=\left\{\begin{array}{lll}b_{x} & b_{y} & b_{z}\end{array}\right\}^{T}, \quad\left\{\sigma^{\prime}\right\}=$ $\left\{\begin{array}{llllll}\sigma_{x}^{\prime} & \sigma_{y}^{\prime} & \sigma_{z}^{\prime} & \tau_{y z} & \tau_{x z} & \tau_{x y}\end{array}\right\}^{T}$ and $\{\sigma\}=\left\{\sigma^{\prime}\right\}+\{m\} p_{f}$ are unit volume force, effective stress, and total stress, respectively. $\{m\}=\left[\begin{array}{llllll}1 & 1 & 1 & 0 & 0 & 0\end{array}\right]^{T}$. Differential operator $[L]$ is defined as

$$
[L]=\left[\begin{array}{cccccc}
\frac{\partial}{\partial \widehat{x}} & 0 & 0 & 0 & \frac{\partial}{\partial \widehat{z}} & \frac{\partial}{\partial \widehat{y}} \\
0 & \frac{\partial}{\partial \widehat{y}} & 0 & \frac{\partial}{\partial \widehat{z}} & 0 & \frac{\partial}{\partial \widehat{x}} \\
0 & 0 & \frac{\partial}{\partial \widehat{y}} & \frac{\partial}{\partial \widehat{y}} & \frac{\partial}{\partial \widehat{x}} & 0
\end{array}\right]^{T} .
$$

The continuity equation of fluid motion in saturated soils in frequency domain is as follows:

$$
-k p_{f, i i}+\omega^{2} k \rho_{f} u_{i, j}+k \rho_{f} b_{i, j}+i \omega u_{i, j}+\frac{i \omega \phi p_{f}}{K_{f}}=0,
$$

where $k$ and $K_{f}$ are the permeability coefficient of soil and the bulk modulus of fluid, respectively.

For isotropic saturated soils, effective stress, strain, and displacement vector satisfy Hooke's law:

$$
\left\{\sigma^{\prime}\right\}=[D]\{\varepsilon\}=[D][L]\{u\},
$$

where $[D]$ is the elastic matrix of saturated soil material. 
According to equation (3), in the scaled curvilinear coordinate system $(\xi, \eta, \zeta)$ shown in Figure 2 , the differential operator $[L]$ is expressed as

$$
\begin{aligned}
& {[L]=\left[b^{1}\right] \frac{\partial}{\partial \xi}+\frac{1}{\xi}\left(\left[b^{2}\right] \frac{\partial}{\partial \eta}+\left[b^{3}\right] \frac{\partial}{\partial \zeta}\right),} \\
& {\left[b^{1}\right]=\frac{g^{\xi}}{|J|}\left[\begin{array}{cccccc}
n_{x}^{\xi} & 0 & 0 & 0 & n_{z}^{\xi} & n_{y}^{\xi} \\
0 & n_{y}^{\xi} & 0 & n_{z}^{\xi} & 0 & n_{x}^{\xi} \\
0 & 0 & n_{z}^{\xi} & n_{y}^{\xi} & n_{x}^{\xi} & 0
\end{array}\right]^{T},} \\
& {\left[b^{2}\right]=\frac{g^{\eta}}{|J|}\left[\begin{array}{llllll}
n_{x}^{\eta} & 0 & 0 & 0 & n_{z}^{\eta} & n_{y}^{\eta} \\
0 & n_{y}^{\eta} & 0 & n_{z}^{\eta} & 0 & n_{x}^{\eta} \\
0 & 0 & n_{z}^{\eta} & n_{y}^{\eta} & n_{x}^{\eta} & 0
\end{array}\right]^{T},} \\
& {\left[b^{3}\right]=\frac{g^{\zeta}}{|J|}\left[\begin{array}{llllll}
n_{x}^{\zeta} & 0 & 0 & 0 & n_{z}^{\zeta} & n_{y}^{\zeta} \\
0 & n_{y}^{\zeta} & 0 & n_{z}^{\zeta} & 0 & n_{x}^{\zeta} \\
0 & 0 & n_{z}^{\zeta} & n_{y}^{\zeta} & n_{x}^{\zeta} & 0
\end{array}\right]^{T},}
\end{aligned}
$$

where $n_{x}^{\xi}, n_{y}^{\xi}, n_{z}^{\xi}, n_{x}^{\eta}, n_{y}^{\eta}, n_{z}^{\eta}, n_{x}^{\zeta}, n_{y}^{\zeta}$, and $n_{z}^{\zeta}$ are the components of the outer surface unit normal $\left(\mathbf{n}^{\xi}, \mathbf{n}^{\eta}\right.$ and $\left.\mathbf{n}^{\zeta}\right)$ of the bottom $S^{e}$ shown in Figure 2, respectively.

Equation (8) shows that $\left[b^{1}\right],\left[b^{2}\right]$, and $\left[b^{3}\right]$ are independent of the radial coordinates $\xi$ and satisfy the following formula:

$$
\left(|J|\left[b^{2}\right]\right)_{\eta}+\left(|J|\left[b^{3}\right]\right)_{\zeta}=-2|J|\left[b^{1}\right] .
$$

In the Cartesian coordinates $(\hat{x}, \hat{y}, \widehat{z})$, the tangential force of a surface with a normal direction of $\mathbf{n}=\mathbf{n}_{x} i+\mathbf{n}_{y} j+$ $\mathbf{n}_{z} k$ is

$$
\{\tau\}=\left\{\begin{array}{c}
\tau_{x} \\
\tau_{y} \\
\tau_{z}
\end{array}\right\}=\left[\begin{array}{cccccc}
n_{x} & 0 & 0 & 0 & n_{z} & n_{y} \\
0 & n_{y} & 0 & n_{z} & 0 & n_{x} \\
0 & 0 & n_{z} & n_{y} & n_{x} & 0
\end{array}\right]\{\sigma\} .
$$

From equations (3), (8), and (10), it can be seen that the tangential force on the surface $(\eta, \zeta),(\zeta, \xi)$, and $(\xi, \eta)$ of any given value $\xi, \eta$, and $\zeta$ is as follows:

$$
\begin{aligned}
& \left\{t^{\xi}\right\}=\frac{|J|}{g^{\xi}}\left[b^{1}\right]^{T}\{\sigma\}, \\
& \left\{t^{\eta}\right\}=\frac{|J|}{g^{\eta}}\left[b^{2}\right]^{T}\{\sigma\}, \\
& \left\{t^{\zeta}\right\}=\frac{|J|}{g^{\zeta}}\left[b^{3}\right]^{T}\{\sigma\} .
\end{aligned}
$$

For the computational element domain $V^{e}$ shown in Figure 2, the displacement and pore pressure of discrete nodes are expressed by a shape function similar to the coordinates of nodes on the bottom boundary $S^{e}$ of $\xi=1$. Then, on a surface $S^{\xi}$ with any radial coordinate $\xi$, displacement and pore pressure can be expressed as

$$
\begin{gathered}
\{u\}=\{u(\xi, \eta, \zeta)\}=\left[N^{u}(\eta, \zeta)\right]\{u(\xi)\}, \\
\left\{p_{f}\right\}=\left\{p_{f}(\xi, \eta, \zeta)\right\}=\left[N^{p}(\eta, \zeta)\right]\left\{p_{f}(\xi)\right\} .
\end{gathered}
$$

From equations (7), (8), and (12), it can be seen that the effective stress in the scaled curvilinear coordinates is as follows:

$$
\begin{aligned}
\left\{\sigma^{\prime}\right\} & =[D]\left(\left[b^{1}\right]^{T}\left\{u_{\xi}\right\}+\frac{1}{\xi}\left(\left[b^{2}\right]^{T}\left\{u_{\eta}\right\}+\left[b^{3}\right]^{T}\left\{u_{\zeta}\right\}\right)\right) \\
& =[D]\left(\left[B_{u}^{1}\right]\{u(\xi)\}_{\xi}+\frac{1}{\xi}\left[B_{u}^{2}\right]\{u(\xi)\}\right), \\
\left\{\sigma_{\xi}^{\prime}\right\} & =[D]\left(\left[B_{u}^{1}\right]\{u(\xi)\}_{\xi \xi}+\frac{1}{\xi}\left[B_{u}^{2}\right]\{u(\xi)\}_{\xi}-\frac{1}{\xi^{2}}\left[B_{u}^{2}\right]\{u(\xi)\}\right),
\end{aligned}
$$

where $\left[B_{u}^{1}\right]^{T}=\left[b^{1}\right]\left[N^{u}\right]$ and $\left[B_{u}^{2}\right]=\left[b^{2}\right]\left[N^{u}\right]_{, \eta}+\left[b^{3}\right]\left[N^{u}\right]_{, \zeta}$. Therefore, it can be concluded that $\left[B_{u}^{1}\right]$ and $\left[B_{u}^{2}\right]$ are only functions of circumferential coordinates, independent of radial coordinates $\eta$, $\zeta$.

By using equations (3), (8), and (12), the differential equations (4) and (6) of saturated soil consolidation in Cartesian coordinate system $(\widehat{x}, \hat{y}, \widehat{z})$ can be transformed into the scaled curvilinear coordinates, and it can be expressed as follows:

$$
\left[b^{1}\right]^{T}\left(\left\{\sigma_{\xi}^{\prime}\right\}+\{m\} p_{f, \xi}\right)+\frac{1}{\xi}\left(\left[b^{2}\right]^{T}\left(\left\{\sigma_{\eta}^{\prime}\right\}+\{m\} p_{f, \eta}\right)+\left[b^{3}\right]^{T}\left(\left\{\sigma_{\zeta}^{\prime}\right\}+\{m\} p_{f, \zeta}\right)\right)+\rho \omega^{2}\{u\}+\rho\{b\}=0
$$




$$
\begin{aligned}
- & k\{m\}^{T}\left(\left[b^{1}\right] \frac{\partial}{\partial \xi}\left(\left[b^{1}\right]^{T} \frac{\partial}{\partial \xi}+\frac{1}{\xi}\left(\left[b^{2}\right]^{T} \frac{\partial}{\partial \eta}+\left[b^{3}\right]^{T} \frac{\partial}{\partial \zeta}\right)\right)+\frac{1}{\xi}\left[b^{2}\right]^{T} \frac{\partial}{\partial \eta}\left(\left[b^{1}\right]^{T} \frac{\partial}{\partial \xi}+\frac{1}{\xi}\left(\left[b^{2}\right]^{T} \frac{\partial}{\partial \eta}+\left[b^{3}\right]^{T} \frac{\partial}{\partial \zeta}\right)\right)\right. \\
& \left.+\left[b^{3}\right]^{T} \frac{\partial}{\partial \zeta}\left(\left[b^{1}\right]^{T} \frac{\partial}{\partial \xi}+\frac{1}{\xi}\left(\left[b^{2}\right]^{T} \frac{\partial}{\partial \eta}+\left[b^{3}\right]^{T} \frac{\partial}{\partial \zeta}\right)\right)\right)\{m\} p_{f}+\omega^{2} k \rho_{f}\{m\}^{T}\left(\left[b^{1}\right]^{T}\left\{u_{\xi}\right\}+\frac{1}{\xi}\left(\left[b^{2}\right]^{T}\left\{u_{\eta}\right\}+\left[b^{3}\right]^{T}\left\{u_{\zeta}\right\}\right)\right) \\
& \left.\left.+k \rho_{f}\{m\}^{T}\left[b^{1}\right]^{T}\left\{b_{\xi}\right\}\right)+\frac{1}{\xi}\left(\left[b^{2}\right]^{T}\left\{b_{\eta}\right\}+\left[b^{3}\right]^{T}\left\{b_{\zeta}\right\}\right)\right)+i \omega\{m\}^{T}\left(\left[b^{1}\right]^{T}\left\{u_{\xi}\right\}+\frac{1}{\xi}\left(\left[b^{2}\right]^{T}\left\{u_{\eta}\right\}+\left[b^{3}\right]^{T}\left\{u_{\zeta}\right\}\right)\right)+\frac{i \omega \phi p_{f}}{K}=0 .
\end{aligned}
$$

\section{Application of Weighted Residual Method}

Galerkin weighted residual method is adopted in computing unit domain $V^{e}$. For convenience of expression, the superscript $e$ of the presentation unit is omitted. Interpolated shape functions of the same form as the displacement of discrete nodes are chosen for the weight function, such as $\left\{w^{u}\right\}=\left\{w^{u}(\xi, \eta, \zeta)\right\}=\left[N^{u}(\eta, \zeta)\right]\left\{w^{u}(\xi)\right\}$. For the dynamic equilibrium equation (12) of saturated consolidation soil, it can be obtained by the Galerkin method in the computational domain:

$$
\begin{aligned}
& \int_{V}\left(\{w\}^{T}\left[b^{1}\right]^{T}\left(\left\{\sigma_{\xi}^{\prime}\right\}+\{m\} p_{f, \xi}\right) \mathrm{d} V+\int_{V} \frac{1}{\xi}\{w\}^{T}\left(\left[b^{2}\right]^{T}\left(\left\{\sigma_{\eta}^{\prime}\right\}+\{m\} p_{f, \eta}\right)+\left[b^{3}\right]^{T}\left(\left\{\sigma_{\zeta}^{\prime}\right\}+\{m\} p_{f, \zeta}\right)\right) \mathrm{d} V\right. \\
& \left.\quad+\rho \omega^{2} \int_{V}\{w\}^{T}\{u\} \mathrm{d} V+\rho \int_{V}\{w\}^{T}\{b\}\right) \mathrm{d} V=0
\end{aligned}
$$

For the second item in the above equation, by using the partial integral and substituting equations (9) and (11), we can obtain

$$
I=\int_{0}^{1} \xi\left(\int_{\Gamma^{\xi}}\{w\}^{T}\left(\left\{t^{\zeta}\right\} g^{\zeta} \mathrm{d} \eta+\left\{t^{\eta}\right\} g^{\eta} \mathrm{d} \zeta\right)-\int_{S^{\xi}}-2\{w\}^{T}\left[b^{1}\right]^{T}+\left\{w_{\eta}\right\}^{T}\left[b^{2}\right]^{T}+\left\{w_{\zeta}\right\}^{T}\left[b^{3}\right]^{T}\right)\left(\left\{\sigma^{\prime}\right\}+\{m\} p_{f}\right)|J| \mathrm{d} \eta \mathrm{d} \zeta \mathrm{d} \xi
$$

By substituting equation (18) into equation (17), the following equation can be obtained:

$$
\begin{aligned}
& \int_{0}^{1}\left(\xi^{2} \int_{S^{\xi}}\{w\}^{T}\left[b^{1}\right]^{T}\left(\left\{\sigma_{\xi}^{\prime}\right\}+\{m\} p_{f, \xi}\right)|J| \mathrm{d} \eta \mathrm{d} \zeta+\xi \int_{\Gamma^{\xi}}\{w\}^{T}\left(\left\{t^{\zeta}\right\} g^{\zeta} d \eta+\left\{t^{\eta}\right\} g^{\eta} \mathrm{d} \zeta\right)\right. \\
& \quad-\xi \int_{S^{\xi}}\left(\left(-2\{w\}^{T}\left[b^{1}\right]^{T}+\left\{w_{\eta}\right\}^{T}\left[b^{2}\right]^{T}+\left\{w_{\zeta}\right\}^{T}\left[b^{3}\right]^{T}\right)\left\{\sigma^{\prime}\right\}+\{m\} p_{f}\right)|J| \mathrm{d} \eta \mathrm{d} \zeta \\
& +\rho \omega^{2} \xi^{2} \int_{S^{\xi}}\{w\}^{T}\{u\}|J| d \eta d \zeta+\rho \xi^{2} \int_{S^{\xi}}\{w\}^{T}\{b\}|J| \mathrm{d} \eta \mathrm{d} \zeta \mathrm{d} \xi=0 .
\end{aligned}
$$

Then, we substituted $\{w\}^{T}=\{w(\xi)\}^{T}\left[N^{u}\right]^{T}$ into the upper equation, and if equality is established for the upper equation in the whole integral domain, then 


$$
\begin{aligned}
& \{w\}^{T} \xi^{2} \int_{S^{\xi}}\left[N^{u}\right]^{T}\left[b^{1}\right]^{T}\left\{\sigma_{\xi}^{\prime}\right\}|J| \mathrm{d} \eta \mathrm{d} \zeta+\{w\}^{T} \xi^{2} \int_{S^{\xi}}\left[N^{u}\right]^{T}\left[b^{1}\right]^{T}\{m\}\left[N^{p}\right]\left\{p_{f}(\xi)\right\}_{\xi}|J| \mathrm{d} \eta \mathrm{d} \zeta \\
& \quad+\{w\}^{T} \xi \int_{S^{\xi}}\left(2\left[N^{u}\right]^{T}\left[b^{1}\right]^{T}-\left[N^{u}\right]_{\eta}^{T}\left[b^{2}\right]^{T}-\left[N^{u}\right]_{\zeta}^{T}\left[b^{3}\right]^{T}\right)\left\{\sigma^{\prime}\right\}|J| \mathrm{d} \eta \mathrm{d} \zeta \\
& \quad+\{w\}^{T} \xi \int_{S^{\xi}}\left(2\left[N^{u}\right]^{T}\left[b^{1}\right]^{T}-\left[N^{u}\right]_{\eta}^{T}\left[b^{2}\right]^{T}-\left[N^{u}\right]_{\zeta}^{T}\left[b^{3}\right]^{T}\right)\{m\}\left[N^{p}\right]\left\{p_{f}(\xi)\right\}|J| \mathrm{d} \eta \mathrm{d} \zeta \\
& \quad+\{w\}^{T} \xi \int_{\Gamma^{\xi}}\left[N^{u}\right]^{T}\left(\left\{t^{\zeta}\right\} g^{\zeta} \mathrm{d} \eta+\left\{t^{\eta}\right\} g^{\eta} \mathrm{d} \zeta\right)+\{w\}^{T} \omega^{2} \xi^{2} \int_{S^{\xi}}\left[N^{u}\right]^{T} \rho\left[N^{u}\right]\{u(\xi)\}|J| \mathrm{d} \eta \mathrm{d} \zeta \\
& \quad+\{w\}^{T} \rho \xi^{2} \int_{S^{\xi}}\left[N^{u}\right]^{T}\{b\}|J| \mathrm{d} \eta \mathrm{d} \zeta=0 .
\end{aligned}
$$

After multiplying $\left[\{w\}^{T}\right]^{-1}$ at both sides of the upper equation and substituting $\left[B_{u}^{1}\right]$ and $\left[B_{u}^{2}\right]$, we can obtain

$$
\begin{aligned}
& \xi^{2} \int_{S^{\xi}}\left[B_{u}^{1}\right]^{T}\left\{\sigma_{\xi}^{\prime}\right\}|J| \mathrm{d} \eta \mathrm{d} \zeta+\xi^{2} \int_{S^{\xi}}\left[B_{u}^{1}\right]^{T}\{m\}\left[N^{p}\right]\left\{p_{f}(\xi)\right\}_{\xi}|J| \mathrm{d} \eta \mathrm{d} \zeta \\
& \quad+\xi \int_{S^{\xi}}\left(2\left[B_{u}^{1}\right]^{T}-\left[B_{u}^{2}\right]^{T}\right)\left\{\sigma^{\prime}\right\}|J| \mathrm{d} \eta \mathrm{d} \zeta+\xi \int_{S^{\xi}}\left(2\left[B_{u}^{1}\right]^{T}-\left[B_{u}^{2}\right]^{T}\right)\{m\}\left[N^{p}\right]\left\{p_{f}(\xi)\right\}|J| \mathrm{d} \eta \mathrm{d} \zeta \\
& \quad+\omega^{2} \xi^{2} \int_{S^{\xi}}\left[N^{u}\right]^{T} \rho\left[N^{u}\right]\{u(\xi)\}|J| \mathrm{d} \eta \mathrm{d} \zeta+\xi\left\{F^{t}(\xi)\right\}+\xi^{2}\left\{F^{b}(\xi)\right\}=0,
\end{aligned}
$$

where $\left\{F^{t}(\xi)\right\}=\int_{\Gamma^{\xi}}\left[N^{u}(\eta, \zeta)\right]^{T}\left(\left\{t^{\zeta}\right\} g^{\zeta} \mathrm{d} \eta+\left\{t^{\eta}\right\} g^{\eta} \mathrm{d} \zeta\right)$ and $\left\{F^{b}(\xi)\right\}=\rho \int_{S^{\xi}}\left[N^{u}(\eta, \zeta)\right]^{T}\{b\}|J| \mathrm{d} \eta \mathrm{d} \zeta \circ$.
By using equations (13), (14), and (21), the following results can be obtained:

$$
\begin{aligned}
& \xi^{2} \int_{S^{\xi}}\left[B_{u}^{1}\right]^{T}[D]\left[B_{u}^{1}\right]|J| \mathrm{d} \eta \mathrm{d} \zeta\{u(\xi)\}_{\xi \xi}+\xi^{2} \int_{S^{\xi}}\left[B_{u}^{1}\right]^{T}\{m\}\left[N^{p}\right]|J| \mathrm{d} \eta \mathrm{d} \zeta\left\{p_{f}(\xi)\right\}_{\xi} \\
& \quad+\xi \int_{S^{\xi}}\left[B_{u}^{1}\right]^{T}[D]\left[B_{u}^{2}\right]|J| \mathrm{d} \eta \mathrm{d} \zeta\{u(\xi)\}_{\xi}+\xi \int_{S^{\xi}}\left(2\left[B_{u}^{1}\right]^{T}-\left[B_{u}^{2}\right]^{T}\right)[D]\left[B_{u}^{1}\right]|J| \mathrm{d} \eta \mathrm{d} \zeta\{u(\xi)\}_{\xi} \\
& \quad-\int_{S^{\xi}}\left[B_{u}^{2}\right]^{T}[D]\left[B_{u}^{2}\right]|J| \mathrm{d} \eta \mathrm{d} \zeta\{u(\xi)\}+\omega^{2} \xi^{2} \int_{S^{\xi}}\left[N^{u}\right]^{T} \rho\left[N^{u}\right]|J| \mathrm{d} \eta \mathrm{d} \zeta\{u(\xi)\} \\
& \quad+\xi \int_{S^{\xi}}\left(2\left[B_{u}^{1}\right]^{T}-\left[B_{u}^{2}\right]^{T}\right)\{m\}\left[N^{p}\right]|J| \mathrm{d} \eta \mathrm{d} \zeta\left\{p_{f}(\xi)\right\}+\xi\left\{F^{t}(\xi)\right\}+\xi^{2}\left\{F^{b}(\xi)\right\}=0 .
\end{aligned}
$$

For the continuity equation of fluid in the consolidation equation of saturated soil, the weight function is expressed by the interpolation function which is the same as the pore pressure. Galerkin's method is used in the calculation domain. Similar to the above deduction, the following can be obtained from equation (16):

$$
\begin{aligned}
\int_{V} & -k\{w\}^{T}\{m\}^{T}\left[b^{1}\right]\left[b^{1}\right]^{T}\{m\} p_{f, \xi \xi}+\frac{1}{\xi}\left(\left[b^{1}\right]\left[b^{2}\right]^{T}\{m\} p_{f, \xi \eta}+\left[b^{1}\right]\left[b^{3}\right]^{T}\{m\} p_{f, \xi \zeta}\right) \\
- & \frac{1}{\xi^{2}}\left(\left[b^{1}\right]\left[b^{2}\right]^{T}\{m\} p_{f, \eta}+\left[b^{1}\right]\left[b^{3}\right]^{T}\{m\} p_{f, \zeta}\right)+\frac{1}{\xi}\left[b^{2}\right]\left(\left[b^{1}\right]_{\eta}^{T}\{m\} p_{f, \xi}+\left[b^{1}\right]^{T}\{m\} p_{f, \xi \eta}\right) \\
+ & \frac{1}{\xi}\left[b^{3}\right]\left(\left[b^{1}\right]_{\zeta}^{T}\{m\} p_{f, \xi}+\left[b^{1}\right]^{T}\{m\} p_{f, \xi \zeta}\right)+\frac{1}{\xi^{2}}\left[b^{2}\right]\left(\left[b^{2}\right]_{\eta}^{T}\{m\} p_{f, \eta}+\left[b^{2}\right]^{T}\{m\} p_{f, \eta \eta}\right)
\end{aligned}
$$




$$
\begin{aligned}
& +\frac{1}{\xi^{2}}\left[b^{2}\right]\left(\left[b^{3}\right]_{\eta}^{T}\{m\} p_{f, \zeta}+\left[b^{3}\right]^{T}\{m\} p_{f, \eta \zeta}\right)+\frac{1}{\xi^{2}}\left[b^{3}\right]\left(\left[b^{2}\right]_{\zeta}^{T}\{m\} p_{f, \eta}+\left[b^{2}\right]^{T}\{m\} p_{f, \eta \zeta}\right) \\
& +\frac{1}{\xi^{2}}\left[b^{3}\right]\left(\left[b^{3}\right]_{\zeta}^{T}\{m\} p_{f, \zeta}+\left[b^{3}\right]^{T}\{m\} p_{f, \zeta \zeta}\right)+\left(i \omega+\omega^{2} k \rho_{f}\right)\{w\}^{T}\{m\}^{T}\left[b^{1}\right]^{T}\{u, \xi\} \\
& +\frac{1}{\xi}\left(\left[b^{2}\right]^{T}\left\{u_{\eta}\right\}+\left[b^{3}\right]^{T}\left\{u_{\zeta}\right\}\right)+k \rho_{f}\{w\}^{T}\{m\}^{T}\left[b^{1}\right]^{T}\left\{b_{\xi}\right\} \\
& +\frac{1}{\xi}\left(\left[b^{2}\right]^{T}\left\{b_{\eta}\right\}+\left[b^{3}\right]^{T}\left\{b_{\zeta}\right\}\right)+\frac{i \omega \phi\{w\}^{T} p_{f}}{K_{f}} \mathrm{~d} V=0 .
\end{aligned}
$$

By using the partial integral and substituting $\left\{w^{p}\right\}^{T}=$ $\left\{w^{p}(\xi)\right\}^{T}\left[N^{p}\right]^{T}$ into the upper equation, the following results can be obtained:

$$
\begin{aligned}
& -\xi^{2}\{w\}^{T} \int_{S^{\xi}}\left[B_{p}^{1}\right]^{T} k\left[B_{p}^{1}\right]|J| \mathrm{d} \eta \mathrm{d} \zeta\left\{p_{f}(\xi)\right\}_{\xi \xi}+\xi\{w\}^{T} \int_{S^{\xi}}\left[B_{p}^{1}\right]^{T} k\left[B_{p}^{2}\right]|J| \mathrm{d} \eta \mathrm{d} \zeta\left\{p_{f}(\xi)\right\}_{\xi} \\
& +\xi\{w\}^{T}\left(\int_{\Gamma^{\xi}}\left[B_{p}^{2 A}\right]^{T} k\left[B_{p}^{1}\right]|J| \mathrm{d} \zeta+\int_{\Gamma^{\xi}}\left[B_{p}^{2 B}\right]^{T} k\left[B_{p}^{1}\right]|J| d \eta\right)\left\{p_{f}(\xi)\right\}_{\xi} \\
& +2 \xi\{w\}^{T} \int_{S^{\xi}}\left[B_{p}^{1}\right]^{T} k\left[B_{p}^{1}\right]|J| \mathrm{d} \eta \mathrm{d} \zeta\left\{p_{f}(\xi)\right\}_{\xi}-\xi\{w\}^{T} \int_{S^{\xi}}\left[B_{p}^{2}\right]^{T} k\left[B_{p}^{1}\right]|J| \mathrm{d} \eta \mathrm{d} \zeta\left\{p_{f}(\xi)\right\}_{\xi} \\
& +\{w\}^{T}\left(\int_{\Gamma^{\xi}}\left[B_{p}^{2 A}\right]^{T} k\left[B_{p}^{2}\right]|J| \mathrm{d} \zeta+\int_{\Gamma^{\xi}}\left[B_{p}^{2 B}\right]^{T} k\left[B_{p}^{2}\right]|J| \mathrm{d} \eta\right)\left\{p_{f}(\xi)\right\} \\
& +\{w\}^{T} \int_{S^{\xi}}\left[B_{p}^{2}\right]^{T} k\left[B_{p}^{2}\right]|J| \mathrm{d} \eta \mathrm{d} \zeta\left\{p_{f}(\xi)\right\}+\xi^{2}\left(i \omega+\omega^{2} k \rho_{f}\right) \int_{S^{\xi}}\left[N^{p}\right]^{T}\{m\}^{T}\left[B_{u}^{1}\right]|J| d \eta d \zeta\{u(\xi)\}_{\xi} \\
& +\xi\left(i \omega+\omega^{2} k \rho_{f}\right)\left(\int_{\Gamma^{\xi}}\left[B_{p}^{2 A}\right]^{T}\left[N^{u}\right]|J| \mathrm{d} \zeta+\left[B_{p}^{2 B}\right]^{T}\left[N^{u}\right]|J| d \eta\right)\{u(\xi)\}-\xi\left(i \omega+\omega^{2} k \rho_{f}\right) \int_{S^{\xi}}\left[B_{p}^{2}\right]^{T}\left[N^{u}\right]|J| \mathrm{d} \eta \mathrm{d} \zeta\{u(\xi)\} \\
& +2 \xi\left(i \omega+\omega^{2} k \rho_{f}\right) \int_{S^{\xi}}\left[N^{p}\right]^{T}\{m\}^{T}\left[B_{u}^{1}\right]|J| \mathrm{d} \eta \mathrm{d} \zeta\{u(\xi)\}+\xi^{2} k \rho_{f} \int_{S^{\xi}}\left[B_{p}^{1}\right]^{T}\left\{b_{\xi}\right\}|J| \mathrm{d} \eta \mathrm{d} \zeta \\
& +2 \xi k \rho_{f}\left[\int_{S^{\xi}}\left[B_{p}^{1}\right]^{T}\{b\}|J| \mathrm{d} \eta \mathrm{d} \zeta+\xi^{2} i \omega \int_{S^{\xi}} \frac{\{w\}^{T} \phi}{K_{f}}\left[N^{p}\right]|J| \mathrm{d} \eta \mathrm{d} \zeta\left\{p_{f}(\xi)\right\}=0,\right.
\end{aligned}
$$

where $\quad\left[B_{p}^{1}\right]=\left[b^{1}\right]^{T}\{m\}\left[N^{p}\right], \quad\left[B_{p}^{2 A}\right]^{T}=\left[N^{p}\right]^{T}\{m\}^{T}\left[b^{2}\right]$, $\left[B_{p}^{2}\right]=\left[b^{2}\right]^{T}\{m\}\left[N^{p}\right]_{\eta}+\left[b^{3}\right]^{T}\{m\}\left[N^{p}\right]_{\zeta}, \quad$ and $\quad\left[B_{p}^{2 B}\right]^{T}=$ $\left[N^{p}\right]^{T}\{m\}^{T}\left[b^{3}\right]$.

The dynamic consolidation equation of saturated soil considering pore water pressure can be obtained by equations (22) and (24). The scaled boundary finite element equation expressed by pore water pressure and displacement is as follows:

$$
\begin{aligned}
& {\left[E_{0}\right] \xi^{2}\{u(\xi)\}_{\xi \xi}+\left[E_{3}\right] \xi^{2}\left\{p_{f}(\xi)\right\}_{\xi}+\left(2\left[E_{3}\right]-\left[E_{4}\right]\right) \xi\left\{p_{f}(\xi)\right\}+\left(2\left[E_{0}\right]-\left[E_{1}\right]+\left[E_{1}\right]^{T}\right) \xi\{u(\xi)\}_{\xi}} \\
& -\left[E_{2}\right]\{u(\xi)\}+\omega^{2}\left[M_{0}\right] \xi^{2}\{u(\xi)\}+\xi^{2}\left\{F^{b}(\xi)\right\}+\xi\left\{F^{t}(\xi)\right\}=0,
\end{aligned}
$$




$$
\begin{aligned}
{\left[E_{5}\right] } & \xi^{2}\left\{p_{f}(\xi)\right\}_{\xi \xi}+\left(2\left[E_{5}\right]-\left[E_{6}\right]+\left[E_{6}\right]^{T}+\left\{F^{t 1}\right\}\right) \xi\left\{p_{f}(\xi)\right\}_{\xi}+\left(-\left[E_{7}\right]+\left\{F^{t 2}\right\}\right)\left\{p_{f}(\xi)\right\}-i \omega\left[M_{1}\right] \xi^{2}\left\{p_{f}(\xi)\right\} \\
& +\left\{F^{t 1}\right\} \xi\left\{p_{f}(\xi)\right\}_{\xi}+\left(-\left[E_{7}\right]+\left\{F^{t 2}\right\}\right) \times\left\{p_{f}(\xi)\right\}-i \omega\left[M_{1}\right] \xi^{2}\left\{p_{f}(\xi)\right\}-\left(i \omega+\omega^{2} k \rho_{f}\right)\left[E_{3}\right]^{T} \xi^{2}\{u(\xi)\}_{\xi} \\
& -\left(i \omega+\omega^{2} k \rho_{f}\right)\left(2\left[E_{3}\right]^{T}-\left[E_{8}\right]+\left\{F^{t 3}\right\}\right) \xi\{u(\xi)\}-k \rho_{f} \xi^{2}\left\{F^{b 1}(\xi)\right\}+2 \xi\left\{F^{b 2}(\xi)\right\} \\
& -\xi\left\{F^{b 3}(\xi)\right\}+\xi\left\{F^{b 4}(\xi)\right\}=0
\end{aligned}
$$

where

$$
\begin{aligned}
& {\left[M_{0}\right]=\int_{S^{\xi}}\left[N^{u}\right]^{T} \rho\left[N^{u}\right]|J| \mathrm{d} \eta \mathrm{d} \zeta,} \\
& {\left[M_{1}\right]=\int_{S^{\xi}} \frac{\left[N^{p}\right]^{T} \phi}{K_{f}\left[N^{p}\right]}|J| \mathrm{d} \eta \mathrm{d} \zeta,} \\
& {\left[E_{0}\right]=\int_{S^{\xi}}\left[B_{u}^{1}\right]^{T}[D]\left(\left[B_{u}^{1}\right]|J| \mathrm{d} \eta \mathrm{d} \zeta\right),} \\
& {\left[E_{1}\right]=\int_{S^{\xi}}\left[B_{u}^{1}\right]^{T}[D]\left[B_{u}^{1}\right]|J| \mathrm{d} \eta \mathrm{d} \zeta,} \\
& {\left[E_{2}\right]=\int_{S^{\xi}}\left[B_{u}^{2}\right]^{T}[D]\left[B_{u}^{2}\right]|J| \mathrm{d} \eta \mathrm{d} \zeta,} \\
& {\left[E_{3}\right]=\int_{S^{\xi}}\left[B_{u}^{1}\right]^{T}\{m\}\left[N^{p}\right]|J| \mathrm{d} \eta \mathrm{d} \zeta,} \\
& {\left[E_{4}\right]=\int_{S^{\xi}}\left[B_{u}^{2}\right]^{T}\{m\}\left[N^{p}\right]|J| \mathrm{d} \eta \mathrm{d} \zeta,} \\
& {\left[E_{5}\right]=\int_{S^{\xi}}\left[B_{p}^{1}\right]^{T} k\left[B_{p}^{1}\right]|J| \mathrm{d} \eta \mathrm{d} \zeta,} \\
& {\left[E_{6}\right]=\int_{S^{\xi}}\left[B_{p}^{2}\right]^{T} k\left[B_{p}^{1}\right]|J| \mathrm{d} \eta \mathrm{d} \zeta,} \\
& {\left[E_{7}\right]=\int_{S^{\xi}}\left[B_{p}^{2}\right]^{T} k\left[B_{p}^{2}\right]|J| \mathrm{d} \eta \mathrm{d} \zeta,}
\end{aligned}
$$

$$
\begin{aligned}
{\left[E_{8}\right] } & =\int_{S^{\xi}}\left[B_{p}^{2}\right]^{T}\left[N^{u}\right]|J| \mathrm{d} \eta \mathrm{d} \zeta, \\
\left\{F^{b 3}(\xi)\right\} & =\int_{S^{\xi}}\left[B_{p}^{2}\right]^{T}\{b\}|J| \mathrm{d} \eta \mathrm{d} \zeta, \\
\left\{F^{b 1}(\xi)\right\} & =\int_{S^{\xi}}\left[B_{p}^{1}\right]^{T}\left\{b_{\xi}\right\}|J| \mathrm{d} \eta \mathrm{d} \zeta, \\
\left\{F^{b 2}(\xi)\right\} & =\int_{S^{\xi}}\left[B_{p}^{1}\right]^{T}\{b\}|J| \mathrm{d} \eta \mathrm{d} \zeta, \\
\left\{F^{b 4}(\xi)\right\} & =\int_{\Gamma^{\xi}}\left[B_{p}^{2 A}\right]^{T}\{b\}|J| \mathrm{d} \zeta+\left[B_{p}^{2 B}\right]^{T}\{b\}|J| \mathrm{d} \eta, \\
\left\{F^{t 1}\right\} & =\int_{\Gamma^{\xi}}\left[B_{p}^{2 A}\right]^{T} k\left[B_{p}^{1}\right]|J| \mathrm{d} \zeta+\int_{\Gamma^{\xi}}\left[B_{p}^{2 B}\right]^{T} k\left[B_{p}^{1}\right]|J| \mathrm{d} \eta, \\
\left\{F^{t 2}\right\} & =\int_{\Gamma^{\xi}}\left[B_{p}^{2 A}\right]^{T} k\left[B_{p}^{2}\right]|J| \mathrm{d} \zeta+\int_{\Gamma^{\xi}}\left[B_{p}^{2 B}\right]^{T} k\left[B_{p}^{2}\right]|J| \mathrm{d} \eta, \\
\left\{F^{t 3}\right\} & =\int_{\Gamma^{\xi}}\left(\left[B_{p}^{2 A}\right]^{T}\left[N^{u}\right]|J| \mathrm{d} \zeta+\left[B_{p}^{2 B}\right]^{T}\left[N^{u}\right]|J| \mathrm{d} \eta\right) .
\end{aligned}
$$

The displacement and pore pressure vectors are expressed by generalized displacement vectors $\{\mathbf{U}\}=\left\{\begin{array}{c}\{u(\xi)\} \\ \left\{p_{f}(\xi)\right\}\end{array}\right\}$.

The second-order differential equations can be obtained by equations (25) and (26):

$$
\begin{aligned}
& \xi^{2}\left[\begin{array}{cc}
{\left[E_{0}\right]} & 0 \\
0 & {\left[E_{5}\right]}
\end{array}\right]\{\mathbf{U}(\xi)\}_{\xi \xi}-\left(\xi^{2}\left[\begin{array}{cc}
\left(i \omega+\omega^{2} k \rho_{f}\right)\left[E_{3}\right]^{T} & 0 \\
0 & -\left[E_{3}\right]
\end{array}\right]-\xi\left[\begin{array}{cc}
2\left[E_{0}\right]-\left[E_{1}\right]+\left[E_{1}\right]^{T} & 0 \\
0 & 2\left[E_{5}\right]-\left[E_{6}\right]+\left[E_{6}\right]^{T}+\left\{F^{t 1}\right\}
\end{array}\right]\right)\{\mathbf{U}(\xi)\}_{\xi} \\
&+\left(\xi^{2}\left[\begin{array}{cc}
\omega^{2}\left[M_{0}\right] & 0 \\
0 & -i \omega\left[M_{1}\right]
\end{array}\right]-\xi\left[\begin{array}{cc}
\left(i \omega+\omega^{2} k \rho_{f}\right)\left(2\left[E_{3}\right]^{T}-\left[E_{8}\right]+\left\{F^{t 3}\right\}\right) & 0 \\
0 & {\left[E_{4}\right]-2\left[E_{3}\right]}
\end{array}\right]-\left[\begin{array}{cc}
{\left[E_{2}\right]} & 0 \\
0 & {\left[E_{7}\right]-\left\{F^{t 2}\right\}}
\end{array}\right]\right)\{\mathbf{U}(\xi)\} \\
&=-\xi^{2}\left\{\begin{array}{c}
\left\{F^{b}(\xi)\right\} \\
-k \rho_{f}\left\{F^{b 1}(\xi)\right\}
\end{array}\right\}-\xi\left\{\begin{array}{c}
\left\{F^{t}(\xi)\right\} \\
-k \rho_{f}\left(2\left\{F^{b 2}(\xi)\right\}-\left\{F^{b 3}(\xi)\right\}+\left\{F^{b 4}(\xi)\right\}\right)
\end{array}\right\} .
\end{aligned}
$$




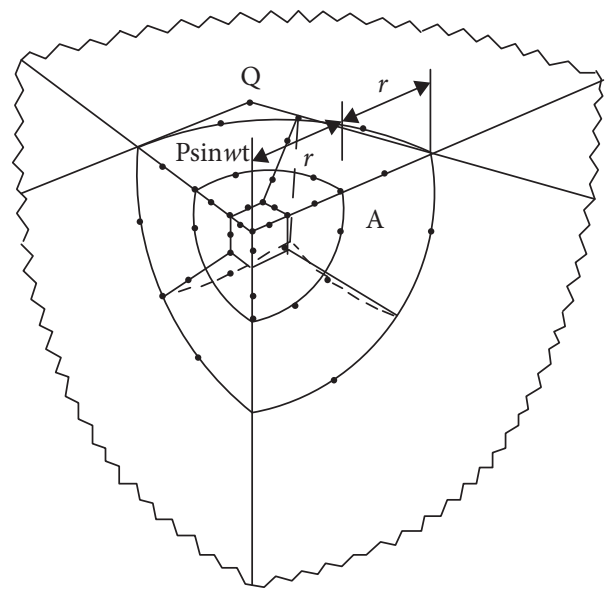

FIgURE 3: SBFEM discretization of $1 / 4$ unbounded half-space.

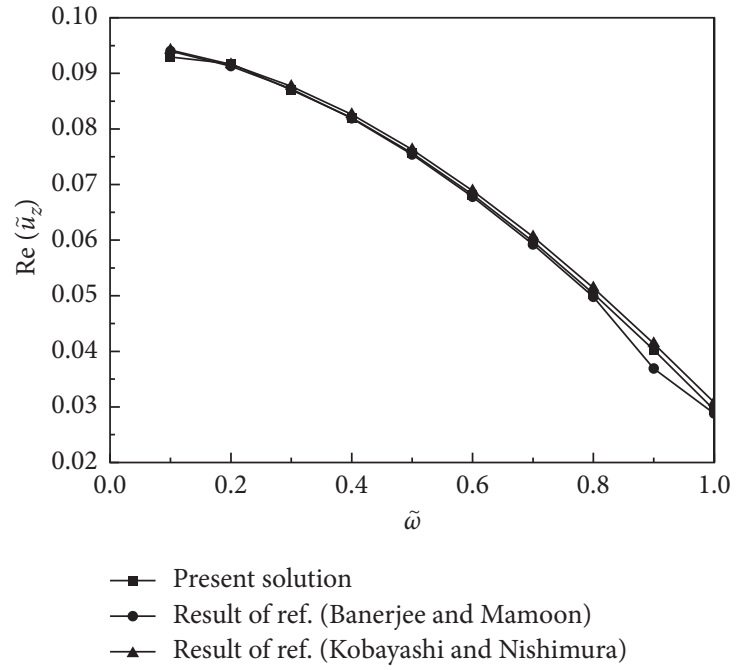

(a)

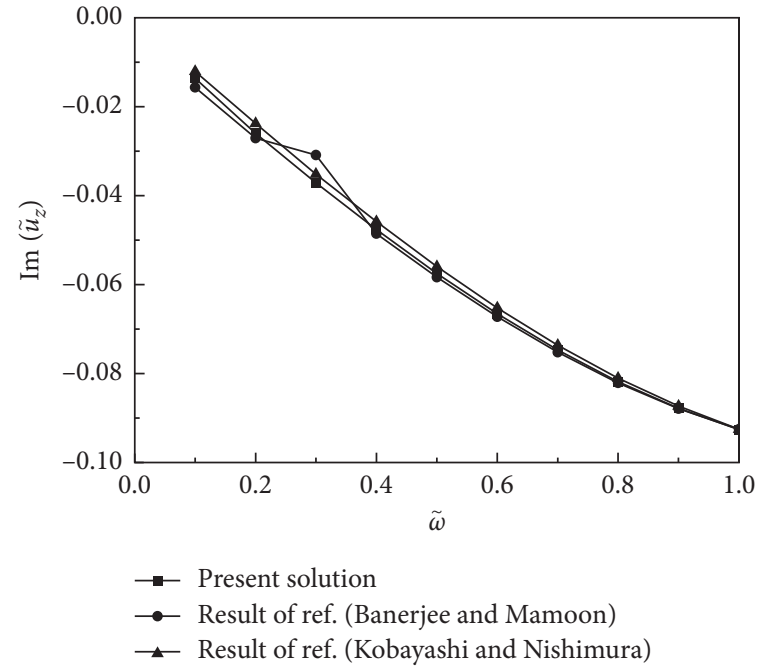

(b)

FIGURE 4: Compared present solutions with the reference's results: (a) real part of vertical displacement and (b) imaginary part of vertical displacement.

\section{Numerical Analysis and Discussion}

The displacement and pore pressure responses of 3D halfspace surface subjected to vertical concentrated harmonic loads are investigated. In the calculation, the saturated soil layer is clay layer, and the relevant parameters in [16] are used. Some soil parameters adopted in the present study are obtained from field investigation and laboratory test, such as $\rho_{s}=2000 \mathrm{~kg} / \mathrm{m}^{3}, \rho_{f}=1000 \mathrm{~kg} / \mathrm{m}^{3}, \phi=0.4, K_{f}=2.0 \times$ $10^{9} \mathrm{~N} / \mathrm{m}^{2}, v=0.4, k=1.0 \times 10^{-9} \mathrm{~m} / \mathrm{s}$, and $\mu=1.0 \times 10^{7} \mathrm{~Pa}$.

Using the method presented in this paper, the $3 \mathrm{D}$ halfspace is divided into two parts. A hemisphere with a radius of $2 \mathrm{R}$ is a structure and the rest is a semi-infinite space. According to symmetry, $1 / 4$ half space is taken for analysis, as shown in Figure 3. Two parts of 3D half-space are analyzed by scaled boundary finite element method. The structure half-space interface is discretized by three 8 -node two-dimensional elements, and the structure part is discretized by eighteen 8 -node two-dimensional elements.

In order to verify the accuracy of this method, if $\rho_{f}=0$ and $n=0$, the two-phase medium degenerates into a singlephase medium. By using the method presented in this paper, the solution of the degenerate 3D linear elastic dynamic Boussinesq problem can be obtained. Figure 4 shows the relationship between vertical displacement $\widetilde{u}_{z}=u_{z} r \mu / P$ and dimensionless frequency $\widetilde{\omega}=\sqrt{\rho / \mu} \omega r$, where $r$ is the horizontal distance between concentrated load $P$ and surface calculation point. It can be seen from Figure 4(a) that the shape of the curve for the present solution is very similar to the solution in the literatures (Banerjee and Mamoon [21] and Kobayashi and Nishimura [22]). The maximum value of the solution in the present solution is 0.092 , and the minimum value is 0.03 , which is consistent with the solution in the literature. And, the difference between them is in the 


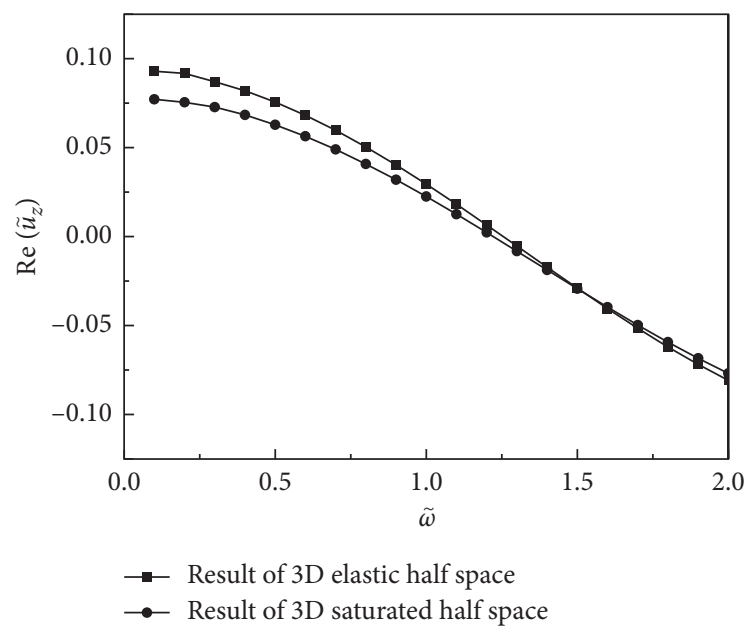

(a)

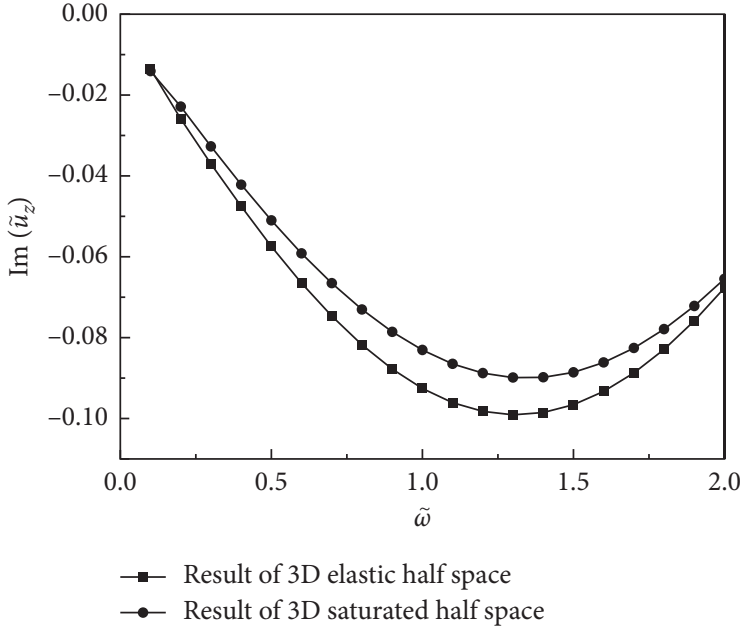

(b)

FIgURE 5: The vertical displacement for the 3D half-space varied with the frequency: (a) real part of vertical displacement and (b) imaginary part of vertical displacement.

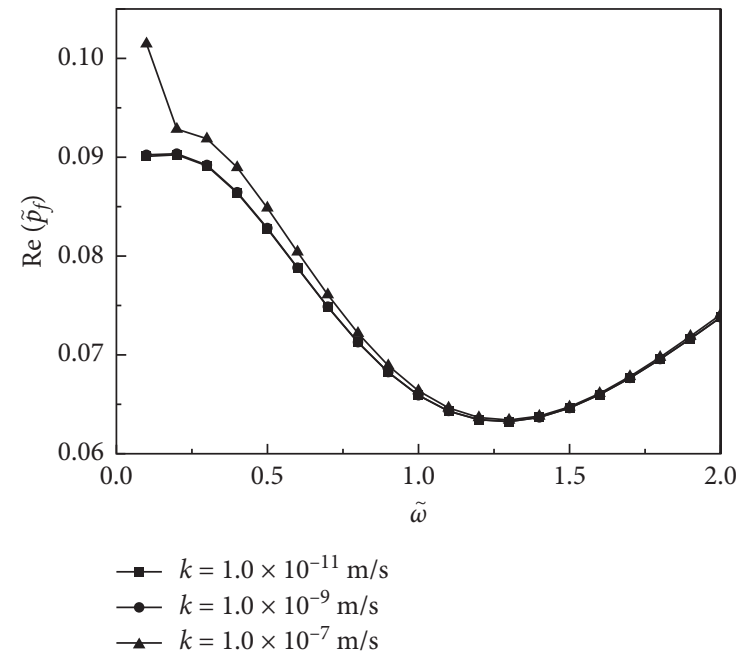

(a)

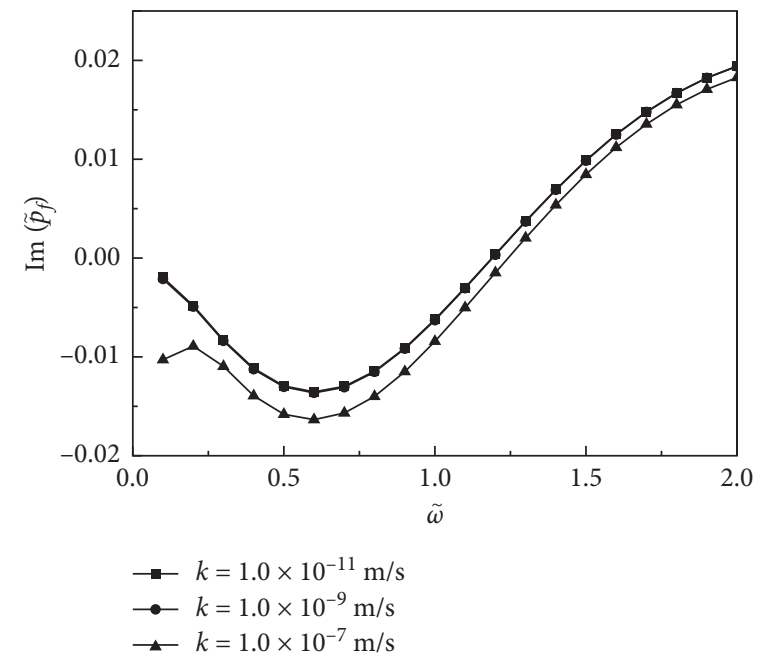

(b)

Figure 6: The pore pressure for the interface between the half spec and structure varied with the frequency: (a) real part of vertical displacement and (b) imaginary part of vertical displacement.

range of 0.002. It can be seen from Figure 4(b) that the solution in present solution has a decreasing trend, and the curve shape is relatively smooth. The maximum value decreased from -0.017 to -0.092 . By comparing the present solution in this paper with that in the literature, it can be concluded that the fluctuation of the solution in present solution is relatively small. The numerical results in this paper are in good agreement with those in literature (Banerjee and Mamoon [21] and Kobayashi and Nishimura [23]), which verifies the accuracy of the proposed method.

Figure 5 shows the relationship between vertical displacement $\widetilde{u}_{z}=u_{z} r \mu / P$ of saturated and elastic soils in 3D half space and dimensionless frequency $\widetilde{\omega}=\sqrt{\rho / \mu} \omega r$. The results show that the dynamic responses of saturated soil and elastic soil are different at different frequencies. It can be seen from the figure that, with the increase of $\widetilde{\omega}$, the real part of the vertical displacement $\widetilde{u}_{z}$ decreases, while the imaginary part of the vertical displacement $\tilde{u}_{z}$ appears at an extreme value near $\widetilde{\omega}=1.3$.

Figure 6 shows the relationship between pore pressure $\widetilde{p}_{f}$ at point $\mathrm{B}$ on the structure-soil interface ( $2 \mathrm{R}$ away from the surface) and dimensionless frequency $\widetilde{\omega}$ at different permeability coefficients $k=1.0 \times 10^{-7} \mathrm{~m} / \mathrm{s}, 1.0 \times 10^{-9} \mathrm{~m} / \mathrm{s}$, and $1.0 \times 10^{-11} \mathrm{~m} / \mathrm{s}$. It can be seen from the figure that the pore pressure amplitude increases with the increase of permeability coefficient of saturated soil.

\section{Conclusion}

In this paper, the scaled boundary element method for single-phase elastic media is extended to the dynamic 
consolidation of 3D saturated soils with water-soil twophase coupling. The application of scaled boundary element method in soil-structure interaction is extended. From the theoretical analysis in this paper, it can be seen that

(1) Not the same as single-phase media, the displacement and stress matrices are not only formed in the scaled boundary finite element equation of saturated soil consolidation but also the pore water pressure effect is coupled in the scaled boundary finite element matrix. According to Biot's theory, the governing differential equation of saturated soil consolidation is solved in Cartesian rectangular coordinate system, and the proportional boundary finite element equation in the form of pore pressure and displacement is obtained.

(2) The scaled boundary of dynamic consolidation of saturated soil also has unique advantages. The dimension of the analytical problem can be reduced by one dimension if only the boundary surface is discretized. In addition, similar to the boundary element method, it can automatically satisfy the radiation conditions at infinite distances. However, the method in this paper does not need to solve the fundamental solution and does not involve singular integrals.

(3) As a semi analytical method, the 3D scaled boundary finite element equation for dynamic consolidation of saturated soils is not only accurate in finite element sense but also convenient in mathematical processing. Especially, in dealing with complex structures and structural nonlinearity, it can simulate two-phase saturated soil-structure dynamic interaction in infinite and finite domain, which has important engineering practical value.

\section{Data Availability}

The data used to support the findings of this study are available from the corresponding author upon request.

\section{Conflicts of Interest}

The authors declare that they have no conflicts of interest.

\section{Acknowledgments}

This work was financially supported by the National Science Foundation of China (no. 5206080257), Key Projects of Natural Science Foundation of Jiangxi Province (nos. 20114BAB206012 and 20181BAB216028), and Jiangxi Province Department of Education Science and Technology Research Project (nos. GJJ12629, GJJ171008, and GJJ11253).

\section{References}

[1] S. L. Chen and H. H. He, "Axisymmetric consolidation of a transversely isotropic single-layered soil," Journal of Shanghai Jiaotong University, vol. 39, no. 5, pp. 760-768, 2005.
[2] J. F. Lu, Y. Si, and J. H. Wang, "Interaction between superstructure, raft and layered viscoelasticsaturated soil," Journal of Shanghai Jiaotong University, vol. 35, no. 4, pp. 489-492, 2001.

[3] J. G. Jiang and H. T. Liang, "Analysis of internal forces of high-risesteel staggered truss under horizontal seismic considering soil-structure interaction," Journal ofRailway Science and Engineering, vol. 8, no. 2, pp. 17-20, 2011.

[4] X. L. Du and J. T. Wang, "Review of studies on the hydrodynamic pressure and its effects on the seismic response of dams," Journal of Hydraulic Engineering, vol. 32, no. 7, pp. 13-21, 2001.

[5] Z. F. Xia, G. L. Ye, J. H. Wang, B. Ye, and F. Zhang, "Fully coupled numerical analysis of repeated shake-consolidation process of earth embankment on liquefiable foundation," Soil Dynamics and Earthquake Engineering, vol. 30, no. 11, pp. 1309-1318, 2010.

[6] C. H. Zhang, Structural-Foundation Dynamic Interaction Problem, pp. 243-266, Hohai University Press, Nanjing, China, 1993.

[7] W. D. A. Smith, "non-reflecting plain boundary for wave propagationproblems," Journal of Computational Physics, vol. 15, no. 4, pp. 492-503, 1974.

[8] B. Engquist and A. Majda, "Absorbing boundary conditions for the numericalsimulationwaves," Mathematics ofComputation, vol. 31, no. 139, pp. 629-651, 1977.

[9] Z. P. Liao, K. L. Huang, and B. P. Yang, "Transmission boundary conditionsfor transient wave analysis," Earthquake Engineering and Engineering Vibration, vol. 2, no. 6, pp. 556-564, 1989.

[10] Z. P. Liao, Z. H. Zhou, and Y. H. Zhang, "Stable implementation of transmitting boundary in numerical simulation of wave motion," Acta Geophysica Sinica, vol. 45, no. 4, pp. 554-568, 2002.

[11] R. G. Keys, "Local Absorbing boundary conditions for acoustic media," Geophysics, vol. 60, no. 5, pp. 892-902, 1985.

[12] J. P. Wolf and C. H. Song, "Doubly asymptotic multidirectional transmitting boundary fordynamic unbounded medium-structure-interactionanalysis," Earthquake Engineering andStructural Dynamics, vol. 24, no. 2, pp. 175-188, 1995.

[13] C. Zhao and S. A. Valliappan, "Dynamic infinite element for three dimensional infinite domain wave problems," International Journal for Numerical Methods in Engineering, vol. 36, pp. 2567-2580, 1992.

[14] N. Khalili, S. Valliappan, J. Tabtabaie-Yazdi, and M. Yazdchi, "1D infinite element for dynamic problems in saturated porous media," Communications in Numerical Methods in Engineering, vol. 13, pp. 727-738, 1997.

[15] G. Bonnet, "Basic singular solutions for a poro-elastic medium in the dynamic range," Journal of the Acoustical Society of America, vol. 82, pp. 1758-1762, 1987.

[16] B. Xu, L. N. Gao, X. Y. Lei, M. Q. Xu, and L. Y. Liu, “Analysis of dynamic interaction between hole embedded in saturated soil and moving loads using 2.5D boundary element method," Journal of Southwest Jiaotong University, vol. 48, no. 4, pp. 659-665, 2013.

[17] M. Yazdchi, N. Khalili, and S. Valliappan, "Non-linear seismic behavior of concrete gravity dams using coupled finite element-boundary element method," International Journal for Numerical Methods in Engineering, vol. 44, no. 1, pp. 101-130, 1999.

[18] Q. K. Du, "The coupling of the natural boundary element and finite element methods for wave equation," Journal of Huaibei Coal Industry Teachers College, vol. 20, no. 2, pp. 1-10, 1999. 
[19] J. P. Wolf and C. M. Song, "Dynamic-stiffness matrix in time domain of unbounded medium by infinitesimal finite-element cell method," Earthquake Engineering andStructural Dynamics, vol. 23, pp. 1181-1198, 1994.

[20] C. M. Song and J. P. Wolf, "The scaled boundary finite-element method alias consistent infinitesimal finite-element cell method," Computer Methods in AppliedMechanics and Engineering, vol. 147, no. 3-4, pp. 329-355, 1997.

[21] P. K. Banerjee and S. M. Mamoon, "A fundamental solution due to a periodic point force in the interior of an elastic halfspace," Earthquake Engineering and Structure Dynamics, vol. 19, no. 1, pp. 91-105, 1990.

[22] S. Kobayashi and N. Nishimura, “Green's tensors for elastic half-space, an application of the boundary integral equation method," Memoirs of the Faculty of Engineering, Kyoto University, vol. 42, pp. 228-241, 1980.

[23] M. Li, H. Zhang, H. Guan, and G. Lin, "Three-dimensional investigation of wave-pile group interaction using the scaled boundary finite element method. Part I: theoretical developments," Ocean Engineering, vol. 63, pp. 174-184, 2013. 